\title{
Transferring patients with Ebola from west Africa to "isolation hospitals" in well resourced countries for treatment
}

\author{
David P Southall honorary medical director, Rhona MacDonald honorary executive director \\ Maternal and Childhealth Advocacy International (MCAI), Laide IV22 2NL, UK
}

The latest predictions regarding the spread of Ebola in west Africa are alarming and leave little time for effective action. ${ }^{12}$ A possible solution would be to transfer Ebola positive patients to suitable "isolation hospitals" in well resourced countries.

Our recent experience in Liberia suggests there is too little time to create and staff enough Ebola treatment units (ETUs) to control the epidemic. ${ }^{3}$ In west Africa, increasing numbers of Ebola positive patients are being turned away and infecting many more people.

It is also becoming increasingly dangerous for national and international staff to work with Ebola positive patients in Liberia and Sierra Leone. Not only is there the risk of infection, but hospitals that can treat other serious illnesses or injuries are poorly functioning.

We suggest that ETUs are urgently opened in well resourced countries. Units could be based in existing isolation hospitals, newly established military field hospitals, or well equipped medical ships lying off west Africa.

The evacuation and treatment process must be totally secure. Military transport planes, with international health workers wearing appropriate protective clothing, could be used.

Patients are more likely to survive in well resourced settings. ETUs in west Africa have reducing numbers of health workers and high dependency care is unavailable. Internationally based
ETUs could provide more effective care, including blood and blood product infusions, accurately managed fluid resuscitation, pain control, and high level nursing care.

After recovery, patients could be returned to west Africa or help care for new patients as nursing assistants.

ETUs in well resourced countries would be run by suitably protected and highly trained volunteer doctors and nurses. Their risk of contracting and dying from Ebola would be lower than in west Africa, and working in well resourced environments might encourage more health workers to volunteer.

This suggestion is likely to create political resistance in countries able to provide such a life saving service, but this concept requires urgent debate. The alternative could be one million men, women, and children dying in terrible circumstances over the next 6-12 months.

\section{Competing interests: None declared.}

Full response at: www.bmj.com/content/349/bmj.g5980/rr/773765.

McCarthy M. Ebola is diagnosed in traveler to US. BMJ 2014;349:g5980. (1 October.) WHO. Global alert and response (GAR). 2014. http://who.int/csr/disease/ebola/en/. Maternal and Childhealth Advocacy International. Latest news from MCAI. Ebola in Liberia. 2014. www.mcai.org.uk/\#!news/c2um.

Cite this as: BMJ 2014;349:96250

๑ BMJ Publishing Group Ltd 2014 